RESEARCH HIGHLIGHT

\title{
Vein to artery: the first arteriogenesis in the mammalian
} embryo

Siyeon Rhe ${ }^{1 凶}$ and Joseph C. Wu (D) ${ }^{1,2,3^{凶}}$

(c) CEMCS, CAS 2022

Cell Research (2022) 32:325-326; https://doi.org/10.1038/s41422-022-00629-7

\begin{abstract}
Dissecting how endothelial cells in vasculature acquire arterial characteristics is critical to understanding of arteriogenesis, but the origin of arterial endothelial cells and the arteriogenesis largely remain unknown. In a recent study from Cell Research, Hou et al. present compelling evidence of early and widespread vein-to-artery cell fate conversion in developing embryos using single-cell transcriptomic analysis and lineage tracing with a newly developed venous Cre line.
\end{abstract}

Oxygen-rich blood from the heart is delivered to other tissues through arteries. Arteriogenesis, the formation of arteries, is an important process in mammalian embryogenesis as arteries play a key role in early embryonic development. ${ }^{1}$ A proper arteriogenesis is necessary to build the stable circulatory system that provides adequate nutrients and oxygen to all the cells. ${ }^{2,3}$ While angiogenesis has been extensively researched, arteriogenesis is now also receiving more and more attention in the field of regenerative medicine as arteries can restore impaired circulation more effectively than capillaries. ${ }^{4}$ However, where arterial endothelial cells (ECs) come from remains a mystery.

To elucidate the underlying mechanism of arterial specification, researchers studied the way developmental systems are established from the early nineteenth century. Initially, chick embryos and zebrafish were common animal models as they both provide platforms to track cell lineage within an in vivo system. ${ }^{1,5-7}$ In the zebrafish, the migration of ECs from veins toward arteries has been observed in later organogenesis stages. ${ }^{5}$ With the advancement of lineage tracing techniques, clonal analysis was adapted to study the blood vasculature development in a wider range of model organisms. Using clonal analysis and mouse embryo explant, Red-Horse et al. first showed that coronary arteries arise from angiogenic sprouts of the sinus venosus. ${ }^{8}$ As sinus venosus is a major vein of the heart, this study was the first of its kind that suggested the venous ECs as progenitors of arteriogenesis. ${ }^{8}$ Within last several years, researchers applied single-cell sequencing technologies to further characterize the plasticity of venous ECs in arterial development. Recently, Su et al. analyzed vein-tocoronary artery cell fate switch at the single-cell resolution, which identified the pre-artery cell type that is specified before blood flow. ${ }^{9}$ Therefore, the potential of venous ECs to be reprogrammed into arteries appears to be a common theme. However, the unresolved question is whether the same phenomenon occurs throughout an entire embryo during the early stages of development.
Hou et al. ${ }^{10}$ discovered an answer to this question. To first understand the heterogeneity of vascular ECs (VECs) at early embryogenesis, they collected cells from both mouse embryos and yolk sac from E8.0 to E11.0 and performed single-cell RNA sequencing (scRNA-seq). After identifying the clusters from the embryo proper and the yolk sac, their scores of arteriovenous features were calculated. By focusing on the embryo proper, the sequencing data revealed that the VECs can be categorized into three main groups: major arterial VECs, arterial plexus VECs, and major venous and vascular plexus VECs.

As scRNA-seq loses the original positions of the cells, Hou et al. used pan-arterial reporter lines (Unc $5 b^{\text {tdTomato }}$ and DII4 ${ }^{\text {tdTomato }}$ ) to map the physical locations of the identified clusters. Surprisingly, the results showed that morphologically distinct major vein and venous plexus exhibit molecularly similar transcriptional profiles, whereas major artery and arterial plexus possess distinct transcriptional profiles. Based on this, Hou et al. concluded that vascular beds of embryos present asymmetric characteristics, unlike the symmetrical zonation of adult vessels ${ }^{1,2}$ (Fig. 1).

In order to confirm that their finding is conserved in humans, scRNA-seq was also performed for intra-embryonic blood vessels of human embryos. By integrating human and mouse data, the results showed that VECs of humans can also be classified into three main groups, illustrating how the proposed model is conserved among mammals.

To predict the developmental paths from the primordial vessels to two types of arterial VECs, Hou et al. investigated the direction of cell differentiation paths via trajectory inference. This analysis enabled the study of dynamic changes in gene expression of VECs and revealed two pathways for arterial specification - one for the major arteries and one for the arterial plexus. In early embryogenesis, branching morphogenesis is generally acknowledged to take place. By undergoing branching morphogenesis, the outward growth results in smaller and smaller branches. However, the trajectory analysis unexpectedly showed that the arterial plexus VECs arise from reprogramming of the venous plexus VECs. Using the human embryo transcriptome datasets, Hou et al. showed that these developmental pathways are evolutionarily conserved in humans.

The trajectory analysis transformed a mystery into a hypothesis, which states that arterial cells come from venous cells during early embryogenesis. To validate that venous plexus VECs are the precursors of arterial plexus VECs, the researchers generated a Nr2f2-CrexER mouse line for inducible genetic lineage tracing and

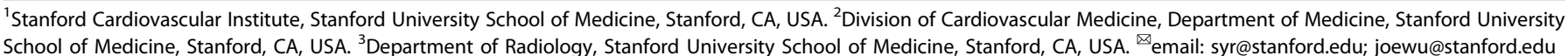

Published online: 14 February 2022 
Conventional model

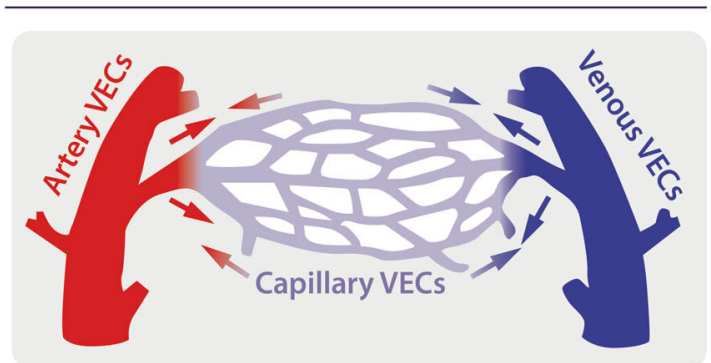

Different properties corresponding to different vascular beds Branching morphogenesis and/or capillary specification
Proposed model

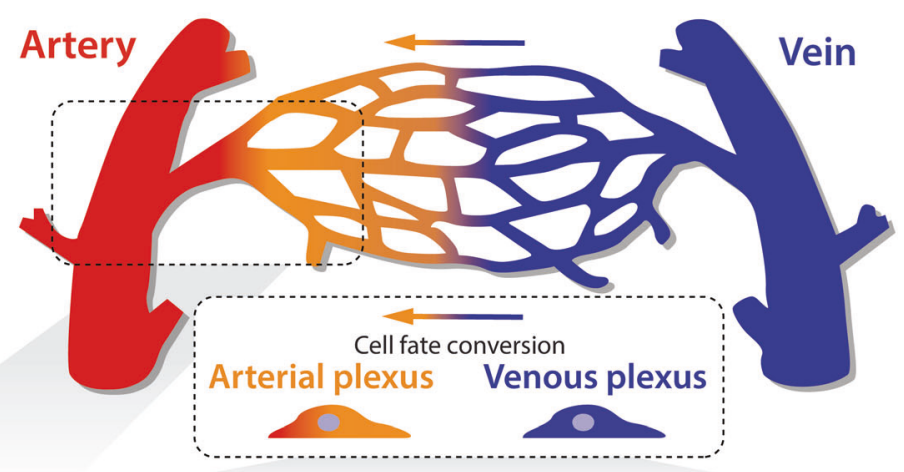

b. Lineage Tracing of venous VECs

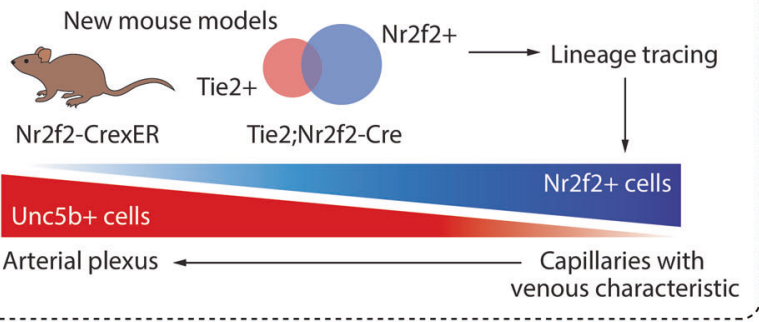

Fig. 1 VECs from capillaries with venous characteristics undergo early cell fate conversion to become arterial plexus, establishing an asymmetric zonation of major artery-arterial plexus-vein and venous plexus. a, b Single-cell transcriptomics reveals VEC heterogeneity (a) and lineage tracing with newly generated mouse lines identifies venous VECs as the progenitors of arterial VECs (b).

a Tie2-Dre;Nr2f2-CrexER mouse line to specifically target venous VECs. Using both constitutive and conditional lineage tracing methods, Hou et al. showed that venous plexus VECs become arterial plexus VECs, and also demonstrated early and widespread venous arterialization during early embryonic development.

This study introduces a new model for embryo arteriogenesis but raises questions at the same time. Based on the plasticity of venous VECs, what is the mechanism that triggers the cell fate conversion process? For therapeutic applications, it would be critical to investigate what environmental cues trigger the fate switch from the capillaries with venous characteristics to arterial plexus VECs. A key environmental cue that may be involved is the forces imparted by blood flow. In zebrafish embryos, it was reported that venous blood flow promotes upstream migration of ECs from capillaries to form arteries. ${ }^{7}$ However, Su et al. showed that the switch from venous to arterial fate occurs in immature coronary plexus, where vessels are not connected to the circulation. ${ }^{9}$ These conflicting results highlight the importance of further investigation into whether the blood flow also regulates the formation of new arteries in early embryogenesis.

The current paper proposes a novel embryonic arteriogenesis model by presenting two sub-populations of arterial VECs using scRNA-seq analysis and dual recombinase-mediated lineage tracing. Future investigation should focus on uncovering how arterial specification is triggered for its potential therapeutic implications. ${ }^{4}$

\section{REFERENCES}

1. Red-Horse, K. \& Siekmann, A. F. Bioessays 41, e1800198 (2019).

2. Ochoa-Espinosa, A. \& Affolter, M. Cold Spring Harb. Perspect. Biol. 4, a008243 (2012).

3. Fujita, M. et al. Development 138, 1705-1715 (2011).

4. Deindl, E. \& Quax, P. H. A. Cells 9, 1439 (2020).

5. Bussmann, J., Wolfe, S. A. \& Siekmann, A. F. Development 138, 1717-1726 (2011).

6. Mikawa, T. \& Fischman, D. A. Proc. Natl. Acad. Sci. USA 89, 9504-9508 (1992).

7. Weijts, B. et al. Nat. Commun. 9, 5314 (2018).

8. Red-Horse, K., Ueno, H., Weissman, I. L. \& Krasnow, M. A. Nature 464, 549-553 (2010).

9. Su, T. et al. Nature 559, 356-362 (2018).

10. Hou, S. et al. Cell Res. https://doi.org/10.1038/s41422-022-00615-z (2022).

\section{COMPETING INTERESTS}

The authors declare no competing interests.

\section{ADDITIONAL INFORMATION}

Correspondence and requests for materials should be addressed to Siyeon Rhee or Joseph C. Wu.

Reprints and permission information is available at http://www.nature.com/ reprints 